Цыганов В. В., Ивщенко Л. И.

Запорожский национальный технический университет. Украина, г. Запорожье

\title{
МЕТОДОЛОГИЯ И ПРАКТИКА ПОВЫШЕНИЯ ИЗНОСОСТОЙКОСТИ ДЕТАЛЕЙ ТРИБОСОПРЯЖЕНИЙ ПРИ МНОГОКОМПОНЕНТНОМ КОНТАКТНОМ ВОЗДЕЙСТВИИ
}

\begin{abstract}
В статье рассмотрен износ деталей трибосопряжений в условиях многокомпонентного динамического нагружения. Основное внимание уделено методике проведения ускоренных испытаний моделей фрикционных пар в условиях, приближенных к реальным условиям эксплуатации трибосопряжений с различными видами нагружения плоских деталей. Представлена методология определения пути трения в трибосопряжении при трехкомпонентном динамическом нагружении. Отмечена связь износостойкости трибосопряжений и структурной однородности поверхностного слоя. Показана возможность управления износостойкостью контактирующих деталей варьированием условиями контакта. [dx.doi.org/10.29010/080.12]
\end{abstract}

Ключевые слова: изнашивание; образеи; многокомпонентное нагружение; трение; поверхностный слой.

Введение и постановка задачи

Основной задачей повышения работоспособности машин и механизмов является исследование и создание оптимальных пар трения, узлов трения, обеспечивающих заданную надежность и долговечность механических систем в установленных условиях эксплуатации. Решение проблемы износостойкости связано с изучением и поиском закономерностей процессов в зоне контактного взаимодействия твердых тел, необходимых для разработки новых методов снижения вредного воздействия трения и изнашивания.

Исследование процессов контактного взаимодействия сопровождается трудностями, связанными с противоречивыми значениями износостойкости трибосопряжений и невозможностью их сопоставления, поскольку они получены различными методами. Как правило, исследования износостойкости фрикционных пар проводятся с использованием машин трения при качении, скольжении образцов или в условиях изнашивания при фреттинге. Полученные при этом результаты не позволяют создать целостную картину процесса изнашивания, отвечающую реальному процессу, так как большая часть трибосопряжений работает в условиях многокомпонентного нагружения с наличием вибраций действующих в различных направлениях, когда детали в процессе эксплуатации испытывают, например, удар и проскальзывание в двух взаимно перпендикулярных направлениях. Данное положение вызвано функциональными взаимными перемещениями в разных направлениях, наличием вибраций или их комбинациями (рис. 1). При этом трибологические характеристики деталей существенно отличаются от тех, которые имеют место при однонаправленном или двухкомпонентном нагружении. Естественно, что в поверхностном слое металла происходят изменения, приводящие к потере работоспособности деталей.

\section{Основная часть}

Большое значение при изучении процессов в зоне контакта приобретают методы ускоренных испытаний пар трения, при которых необходимый объем информации об износостойкости получается в более короткий срок. В связи с этим были разработаны, исследованы и оценены методики и ряд специальных установок позволяющие реализовывать условия сложного нагружения при различных температурах окружающей среды и испытывать образцы в условиях приближенных к реальным [1].

В процессе испытаний основным критерием износостойкости является интенсивность изнашивания, определяемая как отношение величины изношенного слоя поверхности к пути трения, на котором произошло изнашивание. Особое значение приобретает определение пути трения в трибосопряжении при трехкомпонентном динамическом нагружении когда действуют колебания во взаимно перпендикулярных направлениях. Колебания могут иметь нормальное к плоскости контакта на- 


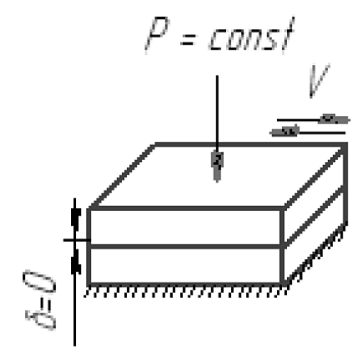

a



б

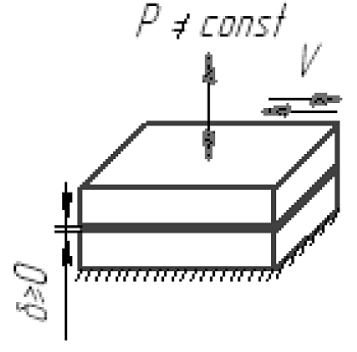

B
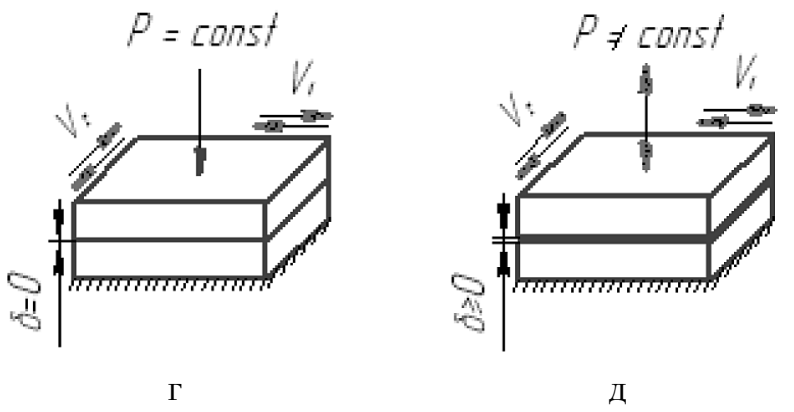

Рис. 1. Схемы нагружения трибосопряжений плоских деталей: а - однокомпонентное при осциллирующем скольжении и постоянной нагрузкой; б - однокомпонентное с периодической ударной нагрузкой; в - двухкомпонентное при осциллирующем скольжении с периодической ударной нагрузкой; г - двухкомпонентное при осциллирующем скольжении

и постоянной нагрузкой; д - трехкомпонентное при осциллирующем скольжении с периодической ударной нагрузкой

правление (ось Z) и тангенциальное направление (в плоскости контакта - ось Х и Y).

Траектория пути трения лежит в плоскости, ориентируемой под определенными углами к осям координат. Величины углов определяются значениями относительных перемещений поверхностей трибосопряжения. Суммарный путь трения точки, располагаемой на линии контакта, обусловлен характером изменения значений по двум осям координат и может иметь форму, отличающуюся от прямой. Однако, учитывая малые амплитуды взаимных перемещений фактом непрямолинейной траектории можно пренебречь [2]. Тогда суммарный путь трения определится исходя из известного выражения:

$$
l^{2}=x^{2}+y^{2},
$$

где $l$ - суммарный путь трения, мм;

$x, y$ - значения смещений по осям координат, мм.

Для установления функциональной зависимости пути трения поверхностей от параметров, задающих направления движения предлагается применить метод моделирования контактного взаимодействия на основе теории физического подобия, с разложением сложного движения на элементарные простые, с последующим их анализом и математическим описанием. При этом будет учитываться комплекс физических величин, определяющих физический процесс взаимодействия контактируемых поверхностей - первая теорема подобия [3].
К физическим величинам можно отнести частоты, амплитуды колебаний поверхностей и время работы трибосопряжения. Для этого подвижный контакт поверхностей рассматривается в виде двух простых движений во взаимно перпендикулярных плоскостях.

Тогда при непрерывном трении путь трения по каждой плоскости определится как

$$
L_{x, y}=A_{x, y} f_{x, y} t
$$

где $A$ - амплитуда колебаний, мм;

$f$ - частота колебаний, мин ${ }^{-1}$;

$t$ - время работы трибосопряжения, мин.

Тогда суммарный путь трения, обусловленный двумя простыми движениями на основании выражения (1), может быть представлен в следующем виде:

$$
L=\sqrt{L_{x}^{2}+k L_{y}^{2}}
$$

где $k$ - коэффициент, учитывающий асинхронность колебаний.

Коэффициент $k$ показывает, на какое количество движений по оси $\mathrm{X}$ приходится движений по оси $\mathrm{Y}$, сопровождающихся трением и перемещением точки по линии контакта. Определяется по отношению:

$$
k=\frac{f_{y}}{f_{x}} .
$$

Кроме того, необходимо также учитывать особенности формирования пути трения по трем осям 
координат при воздействии соответствующих колебаний. В частности колебания по оси $\mathrm{Z}$ могут сопровождаться изменением величины нормального нагружения в трибосопряжении при контакте, что сопровождается перемещениями точки контакта в упругом диапазоне или ударным нагружением при наличии зазора. Сближение деталей вызывает увеличение фактической площади контакта из-за упругой деформации и, следовательно, сила трения в контакте возрастает. При удалении одной детали от другой фактическая площадь контакта уменьшается вплоть до нарушения контакта деталей, сила трения уменьшается, достигая нулевого значения.

Путь трения при ударном нагружении по оси Z приобретает вид:

$$
l_{z}=t A_{z} f_{z}-2 l_{0}
$$

где $l_{0}$ - величина зазора в трибосопряжении, мм.

По трем осям координат путь трения:

$$
\left\{\begin{array}{l}
l_{x}=t A_{x} f_{x} \\
l_{y}=t A_{y} f_{y} \\
l_{z}=t A_{z} f_{z}-2 l_{0} .
\end{array}\right.
$$

На основании выражений (1-5) общий путь трения в трибосопряжении при трехкомпонентном нагружении

$$
\begin{gathered}
L=C \sqrt{\left[\left(t-t_{1}\right) A_{x} f_{x}\right]^{2}+\left[k\left(t-t_{1}\right) A_{y} f_{y}\right]^{2}}= \\
=C\left(t-t_{1}\right) \sqrt{\left(A_{x} f_{x}\right)^{2}+\left(\frac{f_{y}^{2}}{f_{x}} A_{y}\right)^{2}},
\end{gathered}
$$

где $C$ - коэффициент, учитывающий условия контактирования;

$t 1$ - время прерывания контакта при наличии зазора, мин.

Коэффициент условий контактирования включает три основных коэффициента:

$$
C=C_{1} C_{2} C_{3}
$$

где $C_{1}$ - коэффициент фазы гармоники вибрационных смещений:

$C_{2}-$ коэффициент физико-механических свойств контактирующих материалов (упругие и пластические свойства);

$C_{3}$ - коэффициент восстановления нормального и тангенциального импульсов при ударе, который характеризует упругое восстановление деформируемого тела в нормальном и тангенциальном направлениях, является функцией параметров удара [4].

Время прерывания контакта при ударе определяется:

$$
t_{1}=t-t_{2}
$$

где $t_{2}$ - время контакта трибосопряжения при ударе, мин.

Исходя из выражений (2 и 5) время контакта трибосопряжения при ударе определяется:

$$
t_{2}=\frac{l_{z}}{A_{z} f_{z}}=\frac{t A_{z} f_{z}-2 l_{0}}{A_{z} f_{z}}=t-\frac{2 l_{0}}{A_{z} f_{z}} .
$$

С учетом выражений (8-10) выражение (7) принимает окончательный вид:

$L=C_{1} C_{2} C_{3}\left(t-\frac{2 l_{0}}{A_{z} f_{z}}\right) \sqrt{\left(A_{x} f_{x}\right)^{2}+\left(\frac{f_{y}^{2} A_{y}}{f_{x}}\right)^{2}}$

Проведенные исследования позволяют заключить, что величина и распределение износа по контактной поверхности при трении зависит от распределения нормальной динамической нагрузки. Сложный характер нагружения приводит к напряженному состоянию поверхностных слоев материалов трибосопряжения, что сопровождается повышенным износом. Изменение характера нагружения приводит к изменению состояния поверхностного слоя и, как следствие, изменению износостойкости трибосопряжения.

Повышенный износ происходит у деталей с однородным равнопрочным поверхностным слоем. Увеличение поперечных проскальзываний при трении с трехмерным нагружением способствует образованию более однородного поверхностного слоя, снижению его прочности, получению более равномерной микрогеометрии поверхности, которая сопровождается снижением величины и разброса работы выхода электрона по поверхности. Степень этих изменений существенно зависит от химико-физических свойств материалов и условий трения [5-8].

Сложное взаимодействие твердых тел в контактной зоне должно учитываться при построении моделей контактирования и прогнозировании поверхностной прочности материалов при динамических нагрузках и, соответственно, условия проведения испытаний должны быть как можно больше приближены к реальным условиям эксплуатации (однонаправленное скольжение, удар с проскальзыванием, удар с проскальзыванием в двух взаимно перпендикулярных направлениях). Это даст возможность рассмотреть физические основы пластически-деструкционных явлений в зоне контакта трибосопряжений с учетом действия продуктов изнашивания поверхности и разработать рекомендации по обеспечению износостойкого поверхностного слоя контактирующих деталей.

На основании предложенной модели изнашивания, которая учитывает взаимное влияние факто- 
ров нагружения при их совместном действии и объясняет неравномерность износа деталей трибосопряжений за счет различной однородности поверхностного слоя [9], были разработаны следующие конструкторско-технологические рекомендации по повышению износостойкости деталей машин и механизмов:

- конструкторские, направленные на изменение параметров нагружения в трибосопряжении:

1) уменьшение нормального нагружения;

2) исключение ударного нагружения;

3) уменьшение амплитуды поперечных проскальзываний;

4) уменьшение амплитуды продольных проскальзываний;

- технологические, направленные на создание условий контактирования обеспечивающих формирование поверхностного слоя повышенной износостойкости:

1) создание поверхностного слоя с неоднородным структурным состоянием;

2) внедрение в зону контакта свободных макрорадикалов без подачи СОЖ [10];

$3)$ обеспечение оптимальной температуры окружающей среды при эксплуатации трибосопряжения [11, 12].

Управление износостойкостью трибосопряжения предполагает до исследований оценку условно неизменных факторов, установленных на основании предварительного анализа (физико-химические свойства материала деталей трибосопряжения, наличие СОЖ в контакте и др.). Начинаются исследования с оценивания условий кинематического нагружения в трибосопряжении (скольжение, удар, удар и скольжение, удар и скольжение в двух взаимно перпендикулярных направлениях). В процессе исследований производится оценка возможного изменения условий кинематического нагружения в контакте трибосопряжения вследствие износа контактирующих поверхностей при длительных исследованиях. Постоянство условий нагружения обеспечивается корректировкой динамических показателей (амплитудой и частотой продольных перемещений, амплитудой и частотой поперечных перемещений, усилием нормального нагружения, энергией удара).

Кроме того, в процессе исследований производится оценка состояния поверхностного слоя контактирующих поверхностей (трибоспектральный метод, изменение работы выхода электрона по поверхности, величина равновесной шероховатости $[5,6,8-11])$ и при необходимости производится поочередная корректировка условий контактирования (уменьшается амплитуда продольных перемещений, уменьшается амплитуда поперечных перемещений, уменьшается усилие нормального нагружения и энергия удара, оптимизируются величина частоты перемещений и повышенной температуры среды, оптимизируется температура среды, повышается количество свободных макрорадикалов при отсутствии СОЖ). Достижение минимальной однородности и максимальной прочности поверхностного слоя с максимальной работой и дисперсией работы выхода электрона по поверхности, максимальной равновесной шероховатостью подтверждает оптимальность принятых условий контактирования для получения максимальной износостойкости.

\section{Выводы}

Таким образом, для обеспечения повышенной износостойкости трибосопряжений при сложном нагружении необходимо использование комплексного подхода определения взаимосвязи триботехнических и структурных свойств контактирующих материалов при различных видах нагружения. Изменение параметров нагружения достигается уменьшением зазоров в трибосопряжении или путем демпфирования колебаний. Выбор конкретных конструктивно-технологических мероприятий должен производиться с учетом реальных условий эксплуатации трибосопряжения и физики процесса разрушения на основании свойств поверхностного слоя. При этом создаются предпосылки для разработки новых износостойких материалов и покрытий.

\section{Литература}

[1] Ивщенко Л. И. Ускоренные испытания сложнонагруженных деталей трибосопряжений / Л. И. Ивщенко, В. В. Цыганов, В. И. Черный // Вісник двигунобудування. - 2009. - № 1. - С. 150-154.

[2] Кубич В. И. К методике определения пути трения в трибосопряжениях со сложным плоско-параллельным движением / В. И. Кубич, А. О. Щаднев, Л. И. Ивщенко // Проблеми трибології. - 2012. № 1. - С. 45-51.

[3] Браун Э. Д. Моделирование трения и изнашивания в машинах / Э. Д. Браун, Ю. А. Евдокимов, А. В. Чичинадзе. - М.: Машиностроение.- 1982. - 192 с.

[4] Крагельский И. В. Основы расчетов на трение и износ / И. В. Крагельский, М. Н. Добычин, В. С. Комбалов. - М.: Машиностроение.- 1977.$526 \mathrm{c}$.

[5] Ивщенко Л. И. Особенности изнашивания трибосопряжений в условиях трехмерного нагружения / Л. И. Ивщенко, В. В. Цыганов, И. М. Закиев // Трение и износ. - 2011.- Т. № 32, № 1.- С. 500-509.

[6] Ivschenko L. Y. Influence des conditions de chargement dynamique sur la resistance `a l'usure des tribocontacts / L. Y. Ivschenko, V. V. Tsyganov, S. Adjerid // Materiaux \& Techniques. - Volume 101. - 2013. - № 4. P. 403.1-403.7. 
[7] Ивщенко Л. И. Методологические принципы инженерии поверхности деталей ГТУ сложного контактного динамического нагружения / Л. И. Ивщенко, В. В. Цыганов // Технологические системы. 2016. - № 1. - C. 51-59.

[8] Івщенко Л.Й. Вплив тривимірного навантаження на енергетичний стан поверхневого шару деталей ГТД / Л. Й. Івщенко, В. В. Циганов, С. В. Лоскутов, С. В. Сейдаметов // Вісник двигунобудування. 2009. - № 1.-C. 61-65.

[9] Цыганов В. В. Модель структурной организации и разрушения поверхностного слоя деталей при контактировании в условиях многокомпонентного динамического нагружения / В. В. Цыганов, Л. И. Ивщенко / Технологические системы. - 2017. - № 1. C. $84-88$.

[10] Ивщенко Л. И. Особенности трибологических явлений в контакте сопряжений при наличии поли- мера и сложном многокомпонентном нагружении / Л. И. Ивщенко, В. В. Цыганов // Технологические системы. - 2013.- № 4. - С. 16-21.

[11] Ивщенко Л. И. Износостойкость сплава ХТН-61 при отрицательных температурах и сложном динамическом нагружении / Л. И. Ивщенко, В. В. Цыганов, А. Я. Качан // Вестник двигателестроения. 2013. - № 1. - C. 95-103.

[12] Scientific basis of modern technology: experience and prospects. - Monograph: edited by Shalapko J. I. and Dobrzanski L. A. / L. I. Ivshenko, V. V. Tsyganov, J. I. Shalapko // Processes of wear of tribojoints from heat-resistant alloy on cobaltbased at non-stationary thermo-mechanical contact. - Yaremche, 2011. P. 346-354.

Tsyganov V. V., Ivshenko L. I.

Zaporozhye National Technical University. Ukraine, Zaporozhye

\section{METHODOLOGY AND PRACTICE OF INCREASE OF WEARPROOFNESS OF DETAILS OF TRIBOJOINTS AT A MULTICOMPONENT PIN ACTION}

In the article the wear of details of tribojoints is considered in the conditions of multicomponent dynamic ladening. Basic attention is spared to methodology of realization of speed-up tests of models of friction pairs in terms close to the real terms of exploitation of tribojoints with the different types of ladening of flat details. Methodology of determination of way of friction is presented in tribojoint at a three-component dynamic ladening. Connection of wearproofness of tribojoints and structural homogeneity of superficial layer is marked. Management possibility is shown wearproofness of contacting details varying the terms of contact. [dx.doi.org/10.29010/080.12]

Keywords: wear; standard; multicomponent ladening; friction; superficial layer.

\section{References}

[1] Ivschenko L. J. Speed-up tests of difficult ladening details of tribojoints / L. I. Ivshenko, V. V. Tsyganov, V. I. Cherney // Announcer of construction of engines. - 2009. - № 1. - P. 150-154.

[2] Kubech V. I. To of methodology of determination of way of friction in tribojoints with difficult trivial-parallel motion / V. I. Kubech, A. O. Schadnev, L. I. Ivshenko // Problem of tribology. - 2012. - № 1. - P. 45-51.

[3] Braun E. D. Design of friction and wear in the machines / E. D. Braun, U. A. Evdokiev, A. V. Chechenadze. - M.: Engineering.- 1982. $-192 \mathrm{p}$.

[4] Kragelsky I. V. Bases of calculations on a friction and wear / I. V. Kragelsky, M. N. Dobychen, V. S. Kombalov. - M.: Engineering.- 1977.- $526 \mathrm{p}$.

[5] Ivschenko L. J. Features of wear of tribojoints in the conditions of three-dimensional ladening / L. I. Ivshenko, V. V. Tsyganov, I. M. Zakiev // Friction and wear. - 2011.- T. № 32, № 1.- P. 500-509.

[6] Ivschenko L. Y. Influence des conditions de chargement dynamique sur la resistance `a l'usure des tribocontacts / L. Y. Ivschenko, V. V. Tsyganov, S. Adjerid // Materiaux \& Techniques. - Volume 101. - 2013. - № 4. - P. 403.1-403.7.

[7] Ivschenko L. J. Methodological principles engineering surface of details of GTU difficult pin dynamic ladening / L. I. Ivshenko, V. V. Tsyganov // Technological systems. - 2016. - № 1. - P. 51-59. 
[8] Ivschenko L. J. Influence of the three-dimensional loading on the power state of superficial layer of details of GTD / L. I. Ivshenko, V. V. Tsyganov, S. I. Лоскутов, S. I. Seydametov // Announcer of construction of engines. - 2009. № 1.- P. 61-65.

[9] Tsyganov V. V. Model of structural organization and destruction of superficial layer of details at a contact in the conditions of multicomponent dynamic ladening / V. V. Tsyganov, L. I. Ivshenko // Technological systems. - 2017. - № 1. P. 84-88.

[10] Ivschenko L. J. Features of the tribology phenomena are in the contact of interfaces at presence of polymer and difficult multicomponent ladening / L. I. Ivshenko, V. V. Tsyganov // Technological systems. - 2013.- № 4. - P. 16-21.

[11] Ivschenko L. J. Wearproofness of alloy of HTN-61 at negative temperatures and difficult dynamic ladening / L. I. Ivshenko, V. V. Tsyganov, A. Ya. Kachan // Announcer of construction of engines. - 2013. - № 1. - P. 95-103.

[12] Scientific basis of modern technology: experience and prospects. - Monograph: edited by Shalapko J. I. and Dobrzanski L. A. / L. I. Ivshenko, V. V. Tsyganov, J. I. Shalapko // Processes of wear of tribojoints from heat-resistant alloy on cobaltbased at non-stationary thermo-mechanical contact. - Yaremche, 2011. - P. 346-354. 